\title{
Validation of the modified Glasgow Prognostic Score (mGPS) in recurrent ovarian cancer (ROC) - analysis of patients enrolled in the GCIG Symptom Benefit Study (SBS)
}

\section{Background}

The ability to predict the survival of patients with platinum resistant/refractory (PRROC) or "potentially platinum sensitive" (PPSROC) who have been treated with $\geq 3$ lines of chemotherapy (PPS >3) could support physician-patient communication and help guide treatment decisions. The inflammation based modified Glasgow Prognostic Score (mGPS) is predictive of survival in patients with a wide range of advanced cancers, but has not been evaluated in ROC. The aim of this study was to determine the validity of the MGPS in ROC and investigate its associations with health related quality of life (HRQL) and ECOG performance status (PS).

\section{Methods}

All assessments were performed before starting chemotherapy. The mGPS is based on serum levels of $C$ reactive protein (CRP) and albumin, with scores ranging from 0 (least) to 2 (most). HRQL was measured with the EORTC QLQ C-30 and OV-28. $\mathrm{X}^{2}$ tests for trend were used to examine the relationship between HRQL, PS and mGPS. Cox proportional hazards regression was used to assess associations between mGPS, HRQL, clinicopathological factors, and overall survival (OS).

Results

Inflammatory markers were available in 516 of 948 patients in the GCIG SBS. 200 (39\%) had PPS $\geq 3$, $316(61 \%)$ had PRROC. 282 (55\%), 123 (24\%), 111 (22\%) had an mGPS of 0, 1, 2, respectively. Median OS in months was $18.1,9.6$, and 6.6 for mGPS of 0,1 , and 2 respectively. The mGPS was a statistically significant, independent predictor of OS after adjusting for PS and platinum sensitivity $(p<0.001)$. mGPS also remained a significant predictor of OS after adjusting for physical function, role function, global health status, abdominal/GI symptoms, and multiple clinicopathologic factors $(p=0.02)$. Worse PS and higher mGPS were associated with poorer HRQL $(p<0.001)$. Higher mGPS was associated with worse HRQL, independent of PS.

\section{Conclusion}

The MGPS is an independent, significant predictor of OS in ROC after adjusting for HRQL and clinicopathological factors. Higher mGPS is associated with worse HRQL independent of PS. The mGPS is inexpensive, simple to measure, and suitable for use in routine clinical practice, and to both select and stratify participants for clinical trials. 


\section{INTRODUCTION}

Ovarian cancer is the leading cause of gynaecological cancer death in the USA [1]. Most women with ovarian cancer have advanced disease at diagnosis, and are treated with surgery and platinum-based chemotherapy, but over $80 \%$ will experience disease recurrence requiring further therapy. Patients diagnosed with recurrent disease within 6 months of completing platinum-based chemotherapy are classified as having platinum-resistant recurrent ovarian cancer (PRROC).[2]. Patients diagnosed with recurrent disease more than 6 months after completing platinum-based chemotherapy are classified as having "platinum sensitive" recurrent ovarian cancer (PSROC). The overall survival ranges from 12-18 months in PRROC,[3-8] 2-4 years for PSROC, $[2,9,10]$ and gets progressively shorter after multiple lines of chemotherapy.

One of the biggest challenges faced by physicians is selecting patients with ROC who are more likely to benefit from further chemotherapy, and in particular, to identify the subgroup of patients with a very short survival in whom palliative care might be a better option than chemotherapy, in keeping with the ASCO Choosing Wisely campaign. This is particularly important given the discordance in understanding of prognosis between physicians and patients[11]. There is a clear need for a validated method to predict survival that can be used in clinical practice and help frame the important discussion on prognosis and treatment options with patients and families. This will allow patients to make more informed decisions regarding further chemotherapy as well as improve the selection and stratification of patients in clinical trials.

There is mounting evidence that systemic inflammation is associated with clinical decline, increasing pain, and a wide range of systemic symptoms in patients with advanced cancers [12-14]. C-reactive protein( CRP) has been reported to be both prognostic of outcomes, and predictive of response to chemotherapy, in a range of cancers [15]. CRP and albumin are synthesized by the liver and secreted into the circulation with serum levels that are influenced by proinflammatory cytokines such IL1, IL6 and TNF[16, 17]. CRP has also been combined with other markers of systemic inflammation to 
predict prognosis. The best described inflammatory score is the modified Glasgow prognostic score(mGPS)[18], which is calculated from the CRP and serum albumin. The mGPS is predictive of survival in a wide range of advanced cancers [18-20], but to our knowledge has not been validated in ROC.

Health Related Quality of Life (HRQL) is widely accepted to be an important endpoint in cancer clinical trials. HRQL is prognostic in a wide range of advanced cancers [21-26]. There is a good rationale to include $H R Q L$ together with markers of systemic inflammation in a prognostic score. Laird and colleagues recently investigated systemic inflammation and its association with HRQL and PS in patients with advanced cancers, of which a small proportion (6\%) had gynaecologic cancer [27]. They reported that higher mGPS and worse PS were associated with worse HRQOL $(P<.001)$, and that systemic inflammation was associated with worse HRQOL independent of PS.

The aim of this study was to determine the validity of the mGPS in recurrent ovarian cancer and its relationships with HRQL, and performance status (PS).

\section{METHODS}

\section{Trial Design}

The GCIG SBS is a prospective, observational, cohort study that enrolled women from collaborating GCIG clinical trials groups from 11 countries who: were aged 18 years or older, had PRROC prior to starting $2^{\text {nd }}$ or subsequent line chemotherapy or had PPS ROC prior to starting $3^{\text {rd }}$ or subsequent lines of chemotherapy. All participants who had inflammatory markers recorded in their case report form were included in the analyses. Participants completed HRQOL questionnaires before starting chemotherapy. They were required to have a life expectancy of at least 3 months or greater and an ECOG PS of 0-3 for inclusion into the study. 
The specific type, doses, duration, and frequency of administration of chemotherapy were at the discretion of the treating physician, and all supportive treatment and concomitant medications were given as per institutional practice.

The Study was lead and coordinated by the NHMRC Clinical Trials Centre, University of Sydney in collaboration with Australian New Zealand Gynaecological Oncology Group (ANZGOG) and the GCIG Symptom Benefit Committee. The trial was registered on the Australian New Zealand Clinical Trials Registry (ANZCTR: 12607000603415). The Study was performed in accordance to the NHMRC Statement on Ethical Conduct in Research Involving Humans and the Declaration of Helsinki. Ethical approval was obtained at all participating sites, and all participants provided signed, written, informed consent.

\section{Physician assessment of patients}

Physicians assessed participants at baseline and every 3-4 weeks prior to each cycle of chemotherapy. They recorded baseline characteristics, symptoms (symptomatic ascites and cramping abdominal pain), and pre-specified laboratory values including haemoglobin, lactate dehydrogenase (LDH), platelets, CA125, CA125 velocity, ECOG PS, C-reactive protein (CRP), albumin, neutrophil count, and lymphocyte count. Tumour response was assessed at 6-8 week intervals using the same method of assessment throughout the study, at the discretion of the treating physician. Clinical benefit was defined as CA125 response, and/or RECIST response, and/or symptomatic improvement as reported by the treating physician.

\section{Modified Glasgow Prognostic Score (mGPS)}

The serum CRP and albumin concentrations were recorded at baseline before starting chemotherapy. The mGPS is based on serum levels of CRP and albumin, with scores ranging from 0 
to 2 and is calculated as follows: $C R P \leq 10 \mathrm{mg} / \mathrm{L}=0 ; \mathrm{CRP}>10 \mathrm{mg} / \mathrm{L}=1 ; \mathrm{CRP}>10 \mathrm{mg} / \mathrm{L}$ and albumin $<35 \mathrm{~g} / \mathrm{L}=2[14,22]$.

\section{Patient-rated outcomes}

Participants completed HRQL questionnaires at baseline (within 2 weeks before their first cycle of chemotherapy), and then every 3 to 4 weeks before each subsequent cycle of chemotherapy, until disease progression. The questionnaires at baseline included the European Organisation for Research and Treatment of Cancer (EORTC) Core Quality of Life Questionnaire (QLQ C-30) and Ovarian Module (OV28). The QLQ-C30 includes multi-item scales for global health status and five functional domains (physical, role, cognitive, emotional and social) all scored from 0 (worst) to 100 (best); and, six single items for symptoms scored from 0 (least) to 100 (worst). The OV28 includes seven multi-item scales for abdominal/gastrointestinal symptoms (AGIS), peripheral neuropathy, hormonal, body image, attitude to disease/treatment, chemotherapy side effects, and sexuality rated from 0 (worst) to 100 (least).

\section{Statistical Analysis}

Baseline patient characteristics were summarized using mean (standard deviation) and median (range or interquartile range) for continuous variables with normal and skewed distribution, respectively. Categorical variables were expressed as frequencies and percentages. Comparisons of baseline characteristics between patients with and without a calculable mGPS were based on the $\chi^{2}$ test for categorical variables. Comparisons of continuous variables were performed using t-tests or the Wilcoxon rank-sum test for non-normal data.

Progression-free survival (PFS) and overall survival (OS) were analysed using time-to-event methods. Kaplan-Meier curves of PFS and OS by mGPS category were constructed and likewise median PFS/OS 
calculated. Cox proportional hazards regression was used to compute unadjusted hazard ratios (with 95\% confidence intervals) to estimate the effect of mGPS score category on PFS and OS. In addition multivariable Cox regression was performed for OS. The effect of mGPS was first assessed after adjustment for ECOG PS and type of resistance (sensitive vs resistant/refractory); and then after adjustment for HRQL domains and clinicopathological factors. Clinical factors for the latter model were selected using backward elimination; candidate variables were those with $\mathrm{P}<0.05$ in univariable analyses. The final model included physical functioning, role functioning, global health status, abdominal/GI symptoms, haemoglobin, platinum resistance, symptomatic ascites, abdominal cramps, platelets, log CA125, neutrophil: lymphocyte ratio $<5, \mathrm{BMI}$ and CA125 velocity $\geq 5$.

To examine relationships between HRQL, PS, and $m G P S$, we used a series of $X^{2}$ tests for trend in which HRQL subscales were analysed as discrete categories.

All statistical inferences were based on two-sided $p$ values, with values $<0.05$ generally taken to indicate statistical significance. To account for the large number of comparisons depicted in Tables 4 and 5, we used the Bonferroni method to reduce the risk of false positive conclusions by using a more stringent criterion for statistical significance, $\mathrm{P}<0.001$. All analyses were done with $\mathrm{SAS}$ statistical software package, version 9.4 (SAS Institute, Inc., Cary, NC).

\section{RESULTS}

Serum albumin and CRP were available in 516 of 948 (54\%) patients enrolled in the GCIG SBS. Baseline characteristics are shown in Table 1. 200 (39\%) had PPSROC, 316 (61\%) had PRROC. The mGPS score was 0,1 or 2 in 282 (55\%), $123(24 \%)$, and 111 (22\%) patients respectively. The median OS in months was $18.1,9.6$, and 6.6 for mGPS of 0,1 and 2, respectively. The median PFS in months was $5.3,3.7$, and 2.9 for mGPS of 0,1 and 2 , respectively (Table 2). 
In univariable analysis, mGPS was associated with overall survival in the whole group that had inflammatory markers available $(p<0.001)$. In multivariable analysis, mGPS was a statistically significant predictor of OS after adjusting for PS and platinum sensitivity $(p<0.001$, see table 3$)$. The mGPS was also a statistically significant predictor of OS after adjusting for physical function, role function, global health status, abdominal/GI symptoms and the following clinicopathologic factors: haemoglobin, platinum resistance, clinician-rated ascites and abdominal cramps, platelets, CA125, neutrophil: lymphocyte ratio $<5, B M I, C A 125$ velocity $\geq 5$ ( $p=0.024$, see table 3 ).

The associations between HRQL, PS, and mGPS are shown in table 4. Worse PS and higher mGPS were associated with poorer HRQL ( $p<0.001$, see table 4). Lower GHS was associated higher mGPS independent of PS (ie, mGPS 0, 65.3; mGPS 1, 56.1; mGPS 2, 53.1, p<0.001). This association was weaker with role function, physical function $(p<0.023)$, and abdominal/GI symptoms $(p=0.05)$. The associations between symptoms, PS, and mGPS are shown in Table 5. Worse PS and higher mGPS were both associated with more severe symptoms ( $p<0.001$ for fatigue, nausea, pain, dyspnoea, anorexia). Worse nausea/vomiting, pain, dyspnoea and appetite loss were associated with higher mGPS, even though most patients had a good PS of 0 or 1 (e.g. nausea/vomiting: PS 1, mGPS 0 , mean domain score 11.3; mGPS 1, mean domain score 12.3, mGPS 2, mean domain score 23.6, $p<0.001$; see table 5). Higher mGPS was associated with worse HRQL independent of PS. Kaplan-Meier curves showing OS and PFS by mGPS showed those with higher mGPS had shorter OS and PFS (Figure 1).

\section{DISCUSSION}

Our analyses support validity of the MGPS in ROC by demonstrating that it was a significant and independent predictor of OS after adjusting for PS and platinum resistance versus sensitivity. The mGPS remained a significant predictor of OS after adjusting for HRQL and multiple clinicopathologic 
factors including physical function, role function, global health status, abdominal/Gl symptoms as well as haemoglobin, platinum resistance, clinician rated ascites and abdominal cramping, platelet count, CA125, neutrophil: lymphocyte $<5$, Body Mass Index, CA125 velocity $\geq 5$. OS and PFS were significantly longer in women with lower mGPS $(p<0.001)$, that is, those with less systemic inflammation based on baseline levels of serum CRP and albumin.

Worse PS $(<2)$ and higher mGPS were also both associated with worse HRQL. Worse GHS was associated with higher mGPS even after accounting for PS. This was also evident in other functional domains such as role function and physical function. In addition, worse PS and higher mGPS were associated with worse symptoms (fatigue, nausea, pain, dyspnoea, anorexia). Worse nausea/vomiting, pain, dyspnoea and appetite loss were also associated with a higher mGPS, despite $89 \%$ of patients recorded as having a good PS of 0 or 1 . Higher mGPS was also associated with worse HRQL after accounting for PS, and provided additional prognostic information above and beyond that provided by PS.

To the best of our knowledge, this is the first study to test the validity of the mGPS in ROC. Our study analysed over 500 women with recurrent ovarian cancer and included those with both platinum resistant ROC, and with potentially platinum sensitive ROC treated with at least 3 prior lines of chemotherapy. The mGPS was a significant predictor of OS after adjusting for two of the other most clinically relevant prognostic factors in setting: PS and platinum resistance.

We also investigated the relationships between mGPS, HRQL, and PS. There was a positive correlation between worse HRQL and higher mGPS, independent of PS. These findings are consistent with those from a recent study by Laird et al[27] who reported that higher mGPS and lower PS were associated with worse HRQL $(P<.001)$. They also noted that systemic inflammation was associated with worse quality of life independent of PS suggesting a direct relationship between the two. 
The main strengths of our study are its prospective design, analysis plan, and large sample size, and the consistency of our findings regarding the prognostic significance of the mGPS with studies in other tumour types $[18,19,28-30]$. The inclusion of women with ROC and ECOG PS 0-3 is likely to better reflect "real world" practice than a typical clinical trial. We found that worse PS and higher mGPS were associated with worse HRQL, and that worse PS and higher mGPS were associated with worse symptoms. Higher mGPS was associated with worse HRQL independent of PS. A possibole limitation of this study is that not all women enrolled in GCIG SBS had baseline assessment of CRP and albumin as this is not standard of care internationally. However, those who did not have CRP and albumin recorded had similar characteristics to those who did. Further research is needed to assess the validity of the mGPS as a prognostic factor in clinical trials with more homogeneous characteristics at baseline. In addition, consideration should be given to researching interventions designed to modulate the inflammatory response and their effects on symptoms, HRQOL and survival.

PS is widely used to help guide decision making about chemotherapy, but it was developed over 50 years ago and has several limitations. It is subject to bias and there is only a moderate level of agreement between clinician and patient reported PS [31, 32]. For example, PS rated by clinicians was only weakly associated with self-rated PF (Spearman correlation coefficient -0.37 ) in our study. Prognostic scores are not typically used in the clinic to help predict survival of patients with ROC, but a simple, readily available score like the mGPS would be applicable to patients with PRROC as well as in patients with PPSROC who have had multiple lines of prior therapy. There is good evidence that most patients want to be involved in decision making about treatment and involving palliative care services towards the end of life. Physician-patient communication has been identified as an actionable target for reducing overly aggressive care when the prognosis is poor[33]. We have validated the MGPS in women with ROC and shown that combining the MGPS with other prognostic characteristics, such as performance status, and HRQL significantly improves prognostication. The mGPS is a simple, objective score that is readily applicable in routine clinical practice and could 
improve discussion and shared decision making about chemotherapy. It could also be used in clinical trials to improve selection and stratification of participants.

1. Siegel, R.L., K.D. Miller, and A. Jemal, Cancer statistics, 2016. CA Cancer J Clin, 2016. 66(1): p. 7-30.

2. Markman, M., R. Rothman, T. Hakes, B. Reichman, W. Hoskins, S. Rubin, et al., Second-line platinum therapy in patients with ovarian cancer previously treated with cisplatin. Journal of Clinical Oncology, 1991. 9(3): p. 389-393.

3. Thigpen, J.T., J.A. Blessing, H. Ball, S.J. Hummel, and R.J. Barrett, Phase II trial of paclitaxel in patients with progressive ovarian carcinoma after platinum-based chemotherapy: a Gynecologic Oncology Group study. J Clin Oncol, 1994. 12(9): p. 1748-53.

4. Herzog, T.J. and B. Pothuri, Ovarian cancer: a focus on management of recurrent disease. Nature clinical practice Oncology, 2006. 3(11): p. 604-611.

5. Rustin, G., A. Nelstrop, M. Tuxen, and H. Lambert, Defining progression of ovarian carcinoma during follow-up according to CA 125: a North Thames Ovary Group Study. Annals of Oncology, 1996. 7(4): p. 361-364.

6. Blackledge, G., F. Lawton, C. Redman, and K. Kelly, Response of patients in phase II studies of chemotherapy in ovarian cancer: implications for patient treatment and the design of phase II trials. British journal of cancer, 1989. 59(4): p. 650.

7. Gordon, A.N., J.T. Fleagle, D. Guthrie, D.E. Parkin, M.E. Gore, and A.J. Lacave, Recurrent epithelial ovarian carcinoma: a randomized phase III study of pegylated liposomal doxorubicin versus topotecan. Journal of clinical oncology, 2001. 19(14): p. 3312-3322.

8. Davis, A., A.V. Tinker, and M. Friedlander, "Platinum resistant" ovarian cancer: what is it, who to treat and how to measure benefit? Gynecol Oncol, 2014. 133(3): p. 624-31.

9. Markman, M. and M.A. Bookman, Second-line treatment of ovarian cancer. Oncologist, 2000. 5(1): p. 26-35.

10. Gershenson, D.M., J.J. Kavanagh, L.J. Copeland, C.A. Stringer, M. Morris, and J.T. Wharton, Re-treatment of patients with recurrent epithelial ovarian cancer with cisplatin-based chemotherapy. Obstet Gynecol, 1989. 73(5 Pt 1): p. 798-802.

11. Gramling, R., K. Fiscella, G. Xing, M. Hoerger, P. Duberstein, S. Plumb, et al., Determinants of patient-oncologist prognostic discordance in advanced cancer. JAMA oncology, 2016. 2(11): p. 1421-1426.

12. McMillan, D.C., Systemic inflammation, nutritional status and survival in patients with cancer. Current Opinion in Clinical Nutrition \& Metabolic Care, 2009. 12(3): p. 223-226.

13. Fearon, K., Cancer cachexia: developing multimodal therapy for a multidimensional problem. European journal of cancer, 2008. 44(8): p. 1124-1132.

14. McMillan, D.C., An inflammation-based prognostic score and its role in the nutrition-based management of patients with cancer. Proceedings of the Nutrition Society, 2008. 67(03): p. 257-262. 
15. Beer, T.M., A.S. Lalani, S. Lee, M. Mori, K.M. Eilers, J.G. Curd, et al., C-reactive protein as a prognostic marker for men with androgen-independent prostate cancer. Cancer, 2008. 112(11): p. 2377-2383.

16. Diakos, C.I., K.A. Charles, D.C. McMillan, and S.J. Clarke, Cancer-related inflammation and treatment effectiveness. The Lancet Oncology, 2014. 15(11): p. e493-e503.

17. Guthrie, G.J., C.S. Roxburgh, P.G. Horgan, and D.C. McMillan, Does interleukin-6 link explain the link between tumour necrosis, local and systemic inflammatory responses and outcome in patients with colorectal cancer? Cancer treatment reviews, 2013. 39(1): p. 89-96.

18. McMillan, D.C., The systemic inflammation-based Glasgow Prognostic Score: a decade of experience in patients with cancer. Cancer treatment reviews, 2013. 39(5): p. 534-540.

19. Nozoe, T., T. Iguchi, A. Egashira, E. Adachi, A. Matsukuma, and T. Ezaki, Significance of modified Glasgow prognostic score as a useful indicator for prognosis of patients with gastric carcinoma. The American Journal of Surgery, 2011. 201(2): p. 186-191.

20. Proctor, M.J., D.S. Morrison, D. Talwar, S.M. Balmer, C.D. Fletcher, D.S.J. O'Reilly, et al., $A$ comparison of inflammation-based prognostic scores in patients with cancer. A Glasgow Inflammation Outcome Study. European Journal of Cancer, 2011. 47(17): p. 2633-2641.

21. Gotay, C.C., C.T. Kawamoto, A. Bottomley, and F. Efficace, The prognostic significance of patient-reported outcomes in cancer clinical trials. J Clin Oncol, 2008. 26(8): p. 1355-63.

22. Montazeri, A., Quality of life data as prognostic indicators of survival in cancer patients: an overview of the literature from 1982 to 2008. Health Qual Life Outcomes, 2009. 7: p. 102.

23. Quinten, C., C. Coens, M. Mauer, S. Comte, M.A. Sprangers, C. Cleeland, et al., Baseline quality of life as a prognostic indicator of survival: a meta-analysis of individual patient data from EORTC clinical trials. The lancet oncology, 2009. 10(9): p. 865-871.

24. Quinten, C., F. Martinelli, C. Coens, M.A.G. Sprangers, J. Ringash, C. Gotay, et al., A global analysis of multitrial data investigating quality of life and symptoms as prognostic factors for survival in different tumor sites. Cancer, 2014. 120(2): p. 302-311.

25. Grande, G.E., M.C. Farquhar, S.I. Barclay, and C.J. Todd, Quality of life measures (EORTC QLQC3O and SF-36) as predictors of survival in palliative colorectal and lung cancer patients. Palliative and Supportive Care, 2009. 7(03): p. 289-297.

26. Gourgou-Bourgade, S., C. Bascoul-Mollevi, F. Desseigne, M. Ychou, O. Bouche, R. Guimbaud, et al., Impact of FOLFIRINOX compared with gemcitabine on quality of life in patients with metastatic pancreatic cancer: results from the PRODIGE 4/ACCORD 11 randomized trial. J Clin Oncol, 2013. 31(1): p. 23-9.

27. Laird, B.J., M. Fallon, M.J. Hjermstad, S. Tuck, S. Kaasa, P. Klepstad, et al., Quality of Life in Patients With Advanced Cancer: Differential Association With Performance Status and Systemic Inflammatory Response. J Clin Oncol, 2016. 34(23): p. 2769-75.

28. Kishiki, T., T. Masaki, H. Matsuoka, T. Kobayashi, Y. Suzuki, N. Abe, et al., Modified Glasgow prognostic score in patients with incurable stage IV colorectal cancer. The American Journal of Surgery, 2013. 206(2): p. 234-240.

29. Laird, B.J., S. Kaasa, D.C. McMillan, M.T. Fallon, M.J. Hjermstad, P. Fayers, et al., Prognostic Factors in Patients with Advanced Cancer: A Comparison of Clinicopathological Factors and the Development of an Inflammation-Based Prognostic System. Clinical Cancer Research, 2013. 19(19): p. 5456-5464.

30. Li, Q.-Q., Z.-H. Lu, L. Yang, M. Lu, X.-T. Zhang, J. Li, et al., Neutrophil count and the inflammation-based glasgow prognostic score predict survival in patients with advanced gastric cancer receiving first-line chemotherapy. Asian Pacific Journal of Cancer Prevention, 2014. 15(2): p. 945-950.

31. Schnadig, I.D., E.K. Fromme, C.L. Loprinzi, J.A. Sloan, M. Mori, H. Li, et al., Patient-physician disagreement regarding performance status is associated with worse survivorship in patients with advanced cancer. Cancer, 2008. 113(8): p. 2205-2214. 
32. Blagden, S., S. Charman, L. Sharples, L. Magee, and D. Gilligan, Performance status score: do patients and their oncologists agree? British journal of cancer, 2003. 89(6): p. 1022-1027.

33. Robinson, J.D. and R. Jagsi, Physician-Patient Communication-An Actionable Target for Reducing Overly Aggressive Care Near the End of Life. JAMA oncology, 2016. 2(11): p. 14071408.

Table 1. Baseline characteristics

\begin{tabular}{lc}
\hline Characteristic $\mathbf{N}=\mathbf{5 1 6}$ & $\mathbf{N}(\%)$ \\
\hline Age & $12(2)$ \\
\hline $40-49$ & $55(11)$ \\
\hline $50-59$ & $146(28)$ \\
\hline $60-69$ & $174(34)$ \\
$>70$ & $129(25)$ \\
\hline Lines of previous chemotherapy & $104(20)$ \\
\hline 1 & $193(37)$ \\
\hline 2 & $114(22)$ \\
\hline 3 & $105(20)$ \\
\hline $\mathbf{E C O G :}$ & \\
\hline
\end{tabular}

\begin{tabular}{lc}
$\mathbf{0 - 1}$ & $459(89)$ \\
$\geq 2$ & $57(11)$ \\
Cancer-related symptoms present (clinician rated) & $388(75)$ \\
\hline Symptomatic ascites (clinician-rated) & $137(27)$ \\
Crampy abdominal pain or intermittent/incomplete bowel obstruction (clinician- & $219(42)$ \\
rated) &
\end{tabular}




\begin{tabular}{lc}
\hline Response to most recent line: & $67(13)$ \\
\hline CR & $211(41)$ \\
\hline PD & $142(28)$ \\
\hline PR & $81(16)$ \\
\hline SD & $375(73)$ \\
\hline Histopathology: & $141(27)$ \\
\hline Serous & $459(92)$ \\
\hline Other & $451(91)$ \\
\hline Grade: & $44(9)$ \\
\hline High (includes 2 and 3) & \\
\hline Low & \\
\hline
\end{tabular}

Table 2.

\begin{tabular}{cccccc}
\hline mGPS & $\begin{array}{c}\text { Resistant/refractory } \\
\mathbf{N = 3 1 6} \\
\mathbf{n}(\%)\end{array}$ & $\begin{array}{c}\text { Potentially platinum } \\
\text { sensitive } \mathbf{N = 2 0 0} \\
\mathbf{n}(\%)\end{array}$ & $\begin{array}{c}\text { All } \\
\mathbf{N}=\mathbf{5 1 6} \\
\mathbf{n}(\%)\end{array}$ & $\begin{array}{c}\text { mPFS } \\
\text { (months) }\end{array}$ & $\begin{array}{c}\text { mOS } \\
\text { (months) }\end{array}$ \\
\hline $\mathbf{0}$ & $157(50)$ & $125(63)$ & 282 & 5.3 & 18.1 \\
1 & $87(28)$ & $36(18)$ & 123 & 3.7 & 9.6 \\
\end{tabular}


Table 3. Modified Glasgow Prognostic Score as a predictor of overall survival*

\begin{tabular}{ccccccc}
\hline mGPS & HR (95\% Cl) & p- & Overall p- & HR (95\% Cl) & p- & Overall p- \\
& & value & value & & value & value \\
\hline $\mathbf{0}$ & 1 & & $<0.001^{*}$ & 1 & $0.024^{* *}$ \\
1 & $1.58(1.23-$ & $<0.001$ & & $1.27(0.94-1.70)$ & 0.115 & \\
& $2.03)$ & & & & & \\
2 & $2.47(1.90-$ & $<0.001$ & & $1.62(1.14-$ & 0.007 & \\
& $3.21)$ & & $2.29)$ & & \\
\hline
\end{tabular}

*overall cox regression adjusted for ECOG PS and platinum resistance/sensitive

**overall Cox regression adjusted for physical functioning, role functioning, global health status, abdominal/GI symptoms, haemoglobin, platinum resistance, symptomatic ascites, abdominal cramps, platelets, log CA125, neutrophil: lymphocyte $<5$, BMI, CA125 velocity $\geq 5$ 


\begin{tabular}{|c|c|c|c|c|c|c|c|c|c|c|c|c|c|c|}
\hline \multirow[b]{3}{*}{ Domain Score } & \multirow[b]{3}{*}{$E C O G$} & \multicolumn{13}{|c|}{ mGPS } \\
\hline & & \multicolumn{3}{|c|}{0} & \multicolumn{3}{|c|}{1} & \multicolumn{3}{|c|}{2} & \multicolumn{3}{|c|}{ All } & \multirow[b]{2}{*}{$p$-value* } \\
\hline & & $N$ & mean & $s d$ & $N$ & mean & $s d$ & $N$ & mean & $s d$ & $N$ & mean & $s d$ & \\
\hline \multirow[t]{5}{*}{ Global health status score } & 0 & 130 & 65.3 & 20.5 & 27 & 56.2 & 20.0 & 16 & 53.1 & 20.8 & 173 & 62.7 & 20.9 & 0.006 \\
\hline & 1 & 126 & 57.1 & 20.4 & 75 & 57.8 & 23.0 & 67 & 41.8 & 21.4 & 268 & 53.5 & 22.4 & $<.001$ \\
\hline & $2+$ & 14 & 39.3 & 23.7 & 15 & 42.2 & 23.0 & 25 & 42.7 & 22.6 & 54 & 41.7 & 22.6 & 0.670 \\
\hline & All & 270 & 60.1 & 21.5 & 117 & 55.4 & 22.8 & 108 & 43.7 & 21.8 & 495 & 55.4 & 22.8 & $<.001$ \\
\hline & p-value & & $<.001$ & & & 0.133 & & & 0.201 & & & $<.001$ & & \\
\hline \multirow[t]{5}{*}{ Role functional score } & 0 & 131 & 74.7 & 26.9 & 29 & 66.1 & 28.7 & 17 & 57.8 & 30.7 & 177 & 71.7 & 28.0 & 0.009 \\
\hline & 1 & 130 & 64.7 & 29.5 & 76 & 55.7 & 36.1 & 65 & 47.2 & 29.1 & 271 & 58.0 & 32.1 & $<.001$ \\
\hline & $2+$ & 15 & 44.4 & 27.2 & 16 & 50.0 & 35.5 & 23 & 29.0 & 32.7 & 54 & 39.5 & 32.9 & 0.113 \\
\hline & All & 276 & 68.4 & 29.0 & 121 & 57.4 & 34.5 & 105 & 44.9 & 31.3 & 502 & 60.8 & 32.2 & $<.001$ \\
\hline & $p$-value & & $<.001$ & & & 0.104 & & & 0.003 & & & $<.001$ & & \\
\hline \multirow[t]{5}{*}{ Cognitive functional score } & 0 & 130 & 75.5 & 22.2 & 28 & 72.0 & 25.7 & 17 & 73.5 & 19.6 & 175 & 74.8 & 22.5 & 0.539 \\
\hline & 1 & 127 & 73.1 & 24.2 & 76 & 78.5 & 24.9 & 68 & 69.9 & 26.6 & 271 & 73.8 & 25.1 & 0.598 \\
\hline & $2+$ & 14 & 52.4 & 31.3 & 15 & 81.1 & 22.6 & 25 & 64.7 & 29.0 & 54 & 66.0 & 29.5 & 0.381 \\
\hline & All & 271 & 73.2 & 24.1 & 119 & 77.3 & 24.8 & 110 & 69.2 & 26.2 & 500 & 73.3 & 24.8 & 0.357 \\
\hline & $p$-value & & 0.011 & & & 0.200 & & & 0.266 & & & 0.065 & & \\
\hline \multirow[t]{5}{*}{ Physical function score } & 0 & 130 & 78.5 & 18.1 & 29 & 72.1 & 22.6 & 17 & 69.0 & 22.5 & 176 & 76.5 & 19.6 & 0.023 \\
\hline & 1 & 130 & 68.7 & 19.3 & 76 & 65.5 & 23.8 & 68 & 55.4 & 22.4 & 274 & 64.5 & 22.0 & $<.001$ \\
\hline & $2+$ & 15 & 50.2 & 18.0 & 16 & 52.9 & 19.1 & 25 & 42.3 & 28.8 & 56 & 47.4 & 23.8 & 0.245 \\
\hline & All & 275 & 72.3 & 19.9 & 121 & 65.4 & 23.5 & 110 & 54.5 & 25.1 & 506 & 66.8 & 23.1 & $<.001$ \\
\hline & $\mathrm{p}$-value & & $<.001$ & & & 0.011 & & & $<.001$ & & & $<.001$ & & \\
\hline
\end{tabular}




\begin{tabular}{|c|c|c|c|c|c|c|c|c|c|c|c|c|c|c|}
\hline \multirow[b]{3}{*}{ Domain Score } & \multirow[b]{3}{*}{$E C O G$} & \multicolumn{13}{|c|}{$m G P S$} \\
\hline & & \multicolumn{3}{|c|}{0} & \multicolumn{3}{|c|}{1} & \multicolumn{3}{|c|}{2} & \multicolumn{3}{|c|}{ All } & \multirow[b]{2}{*}{$p$-value* } \\
\hline & & $N$ & mean & $s d$ & $N$ & mean & $s d$ & $N$ & mean & $s d$ & $N$ & mean & $s d$ & \\
\hline \multirow[t]{5}{*}{ Emotional functional score } & 0 & 130 & 65.4 & 23.7 & 28 & 66.4 & 26.4 & 17 & 73.0 & 20.5 & 175 & 66.3 & 23.8 & 0.256 \\
\hline & 1 & 128 & 64.9 & 24.0 & 76 & 66.9 & 24.7 & 68 & 57.7 & 27.1 & 272 & 63.7 & 25.2 & 0.097 \\
\hline & $2+$ & 13 & 53.4 & 23.8 & 15 & 67.2 & 26.1 & 25 & 64.0 & 25.3 & 53 & 62.3 & 25.3 & 0.293 \\
\hline & All & 271 & 64.6 & 23.9 & 119 & 66.8 & 25.1 & 110 & 61.5 & 26.2 & 500 & 64.4 & 24.7 & 0.422 \\
\hline & $\mathrm{p}$-value & & 0.281 & & & 0.909 & & & 0.428 & & & 0.212 & & \\
\hline \multirow[t]{5}{*}{ Social functional score } & 0 & 130 & 69.4 & 28.3 & 28 & 67.3 & 28.1 & 17 & 70.6 & 24.0 & 175 & 69.1 & 27.7 & 0.988 \\
\hline & 1 & 128 & 65.4 & 29.4 & 76 & 64.5 & 32.1 & 67 & 56.2 & 30.4 & 271 & 62.9 & 30.6 & 0.063 \\
\hline & $2+$ & 13 & 48.7 & 29.2 & 15 & 66.7 & 28.9 & 25 & 50.7 & 30.2 & 53 & 54.7 & 30.0 & 0.896 \\
\hline & All & 271 & 66.5 & 29.1 & 119 & 65.4 & 30.6 & 109 & 57.2 & 29.9 & 499 & 64.2 & 29.8 & 0.010 \\
\hline & $p$-value & & 0.031 & & & 0.867 & & & 0.042 & & & 0.001 & & \\
\hline \multirow[t]{5}{*}{ Abdominal/Gl } & 0 & 130 & 24.8 & 21.0 & 28 & 32.9 & 21.6 & 17 & 32.2 & 21.7 & 175 & 26.8 & 21.3 & 0.054 \\
\hline & 1 & 129 & 34.1 & 23.7 & 76 & 35.2 & 22.1 & 66 & 45.4 & 23.1 & 271 & 37.1 & 23.5 & 0.003 \\
\hline & $2+$ & 15 & 39.7 & 26.5 & 16 & 34.1 & 20.1 & 24 & 46.6 & 23.7 & 55 & 41.1 & 23.7 & 0.288 \\
\hline & All & 274 & 30.0 & 23.1 & 120 & 34.5 & 21.6 & 107 & 43.6 & 23.3 & 501 & 34.0 & 23.4 & $<.001$ \\
\hline & $p$-value & & $<.001$ & & & 0.793 & & & 0.073 & & & $<.001$ & & \\
\hline
\end{tabular}


Table 5. Relationship between EORTC QLQ C-30 symptoms scales and PS and mGPS

mGPS

\begin{tabular}{|c|c|c|c|c|c|c|c|c|c|c|c|c|c|c|}
\hline \multirow[b]{3}{*}{ Domain Score } & \multirow[b]{3}{*}{$E C O G$} & \multicolumn{13}{|c|}{$m G P S$} \\
\hline & & \multicolumn{3}{|c|}{0} & \multicolumn{3}{|c|}{1} & \multicolumn{3}{|c|}{2} & \multicolumn{3}{|c|}{ All } & \multirow[b]{2}{*}{$p$-value* } \\
\hline & & $N$ & mean & $s d$ & $N$ & mean & $s d$ & $N$ & mean & $s d$ & $N$ & mean & $s d$ & \\
\hline \multirow[t]{5}{*}{ Fatigue } & 0 & 130 & 37.2 & 23.1 & 29 & 43.7 & 20.6 & 17 & 43.1 & 20.0 & 176 & 38.9 & 22.4 & 0.141 \\
\hline & 1 & 130 & 46.3 & 25.1 & 76 & 44.7 & 26.8 & 67 & 61.4 & 24.3 & 273 & 49.6 & 26.2 & $<.001$ \\
\hline & $2+$ & 15 & 73.0 & 24.4 & 16 & 53.8 & 26.4 & 25 & 68.9 & 28.1 & 56 & 65.7 & 27.3 & 0.864 \\
\hline & All & 275 & 43.5 & 25.5 & 121 & 45.7 & 25.4 & 109 & 60.2 & 25.7 & 505 & 47.6 & 26.3 & $<.001$ \\
\hline & $\mathrm{p}$-value & & $<.001$ & & & 0.262 & & & 0.002 & & & $<.001$ & & \\
\hline \multirow[t]{5}{*}{ Nausea / Vomiting } & 0 & 130 & 9.5 & 19.9 & 29 & 9.8 & 19.2 & 17 & 16.7 & 28.3 & 176 & 10.2 & 20.7 & 0.249 \\
\hline & 1 & 130 & 11.3 & 20.0 & 76 & 12.3 & 21.7 & 67 & 23.6 & 25.5 & 273 & 14.6 & 22.5 & $<.001$ \\
\hline & $2+$ & 15 & 36.7 & 36.8 & 16 & 20.8 & 33.6 & 25 & 28.0 & 29.9 & 56 & 28.3 & 32.9 & 0.514 \\
\hline & All & 275 & 11.8 & 21.9 & 121 & 12.8 & 23.0 & 109 & 23.5 & 27.0 & 505 & 14.6 & 23.8 & $<.001$ \\
\hline & $\mathrm{p}$-value & & 0.002 & & & 0.155 & & & 0.188 & & & $<.001$ & & \\
\hline \multirow[t]{5}{*}{ Pain } & 0 & 130 & 26.5 & 27.0 & 29 & 39.7 & 31.6 & 17 & 42.2 & 28.9 & 176 & 30.2 & 28.5 & 0.006 \\
\hline & 1 & 130 & 33.5 & 28.2 & 76 & 40.6 & 31.2 & 68 & 49.8 & 30.9 & 274 & 39.5 & 30.4 & $<.001$ \\
\hline & $2+$ & 14 & 53.6 & 40.9 & 16 & 40.6 & 36.0 & 25 & 39.3 & 34.3 & 55 & 43.3 & 36.4 & 0.270 \\
\hline & All & 274 & 31.2 & 29.0 & 121 & 40.4 & 31.7 & 110 & 46.2 & 31.5 & 505 & 36.7 & 30.8 & $<.001$ \\
\hline & $p$-value & & 0.001 & & & 0.906 & & & 0.611 & & & $<.001$ & & \\
\hline \multirow[t]{5}{*}{ Dyspnoea } & 0 & 128 & 23.2 & 26.6 & 29 & 26.4 & 28.7 & 17 & 35.3 & 34.3 & 174 & 24.9 & 27.9 & 0.097 \\
\hline & 1 & 129 & 25.6 & 30.8 & 75 & 29.8 & 30.8 & 67 & 46.3 & 33.3 & 271 & 31.9 & 32.4 & $<.001$ \\
\hline & $2+$ & 15 & 31.1 & 34.4 & 16 & 33.3 & 36.5 & 25 & 54.7 & 37.1 & 56 & 42.3 & 37.3 & 0.039 \\
\hline & All & 272 & 24.8 & 29.1 & 120 & 29.4 & 30.9 & 109 & 46.5 & 34.6 & 501 & 30.6 & 31.9 & $<.001$ \\
\hline & $\mathrm{p}$-value & & 0.297 & & & 0.464 & & & 0.076 & & & $<.001$ & & \\
\hline
\end{tabular}




\begin{tabular}{|c|c|c|c|c|c|c|c|c|c|c|c|c|c|c|}
\hline \multirow[b]{3}{*}{ Domain Score } & \multirow[b]{3}{*}{$E C O G$} & \multicolumn{13}{|c|}{$m G P S$} \\
\hline & & \multicolumn{3}{|c|}{0} & \multicolumn{3}{|c|}{1} & \multicolumn{3}{|c|}{2} & \multicolumn{3}{|c|}{ All } & \multirow[b]{2}{*}{$p$-value* } \\
\hline & & $N$ & mean & $s d$ & $N$ & mean & $s d$ & $N$ & mean & $s d$ & $N$ & mean & $s d$ & \\
\hline \multirow[t]{5}{*}{ Insomnia } & 0 & 129 & 28.2 & 30.5 & 29 & 37.9 & 30.5 & 17 & 25.5 & 25.1 & 175 & 29.5 & 30.1 & 0.681 \\
\hline & 1 & 130 & 38.2 & 30.8 & 76 & 38.2 & 35.6 & 67 & 47.3 & 31.9 & 273 & 40.4 & 32.6 & 0.090 \\
\hline & $2+$ & 15 & 46.7 & 35.2 & 16 & 39.6 & 37.0 & 25 & 50.7 & 34.9 & 56 & 46.4 & 35.2 & 0.638 \\
\hline & All & 274 & 33.9 & 31.3 & 121 & 38.3 & 34.3 & 109 & 44.6 & 32.5 & 504 & 37.3 & 32.5 & 0.004 \\
\hline & $\mathrm{p}$-value & & 0.002 & & & 0.891 & & & 0.023 & & & $<.001$ & & \\
\hline \multirow[t]{5}{*}{ Appetite loss } & 0 & 130 & 17.7 & 24.3 & 29 & 19.5 & 20.9 & 17 & 27.5 & 31.7 & 176 & 18.9 & 24.6 & 0.148 \\
\hline & 1 & 130 & 25.4 & 31.3 & 76 & 24.1 & 30.6 & 66 & 44.9 & 35.3 & 272 & 29.8 & 33.1 & $<.001$ \\
\hline & $2+$ & 15 & 53.3 & 35.2 & 16 & 47.9 & 40.3 & 25 & 65.3 & 32.6 & 56 & 57.1 & 35.8 & 0.237 \\
\hline & All & 275 & 23.3 & 29.5 & 121 & 26.2 & 31.1 & 108 & 46.9 & 35.9 & 504 & 29.0 & 32.7 & $<.001$ \\
\hline & $p$-value & & $<.001$ & & & 0.009 & & & $<.001$ & & & $<.001$ & & \\
\hline \multirow[t]{5}{*}{ Constipation } & 0 & 130 & 19.7 & 29.8 & 29 & 32.2 & 37.2 & 17 & 31.4 & 34.3 & 176 & 22.9 & 31.9 & 0.043 \\
\hline & 1 & 130 & 27.4 & 33.8 & 75 & 21.3 & 30.8 & 67 & 33.3 & 37.2 & 272 & 27.2 & 34.1 & 0.408 \\
\hline & $2+$ & 15 & 44.4 & 41.1 & 15 & 11.1 & 16.3 & 24 & 27.8 & 38.9 & 54 & 27.8 & 36.5 & 0.263 \\
\hline & All & 275 & 24.7 & 32.9 & 119 & 22.7 & 31.6 & 108 & 31.8 & 36.9 & 502 & 25.8 & 33.6 & 0.123 \\
\hline & $p$-value & & 0.004 & & & 0.029 & & & 0.704 & & & 0.199 & & \\
\hline \multirow[t]{5}{*}{ Diarrhoea } & 0 & 130 & 11.3 & 20.1 & 28 & 14.3 & 21.1 & 17 & 3.9 & 11.1 & 175 & 11.0 & 19.7 & 0.371 \\
\hline & 1 & 128 & 17.2 & 29.9 & 75 & 12.0 & 22.4 & 68 & 22.1 & 32.4 & 271 & 17.0 & 28.8 & 0.414 \\
\hline & $2+$ & 14 & 31.0 & 27.6 & 14 & 11.9 & 21.1 & 25 & 24.0 & 31.2 & 53 & 22.6 & 28.3 & 0.631 \\
\hline & All & 272 & 15.1 & 25.9 & 117 & 12.5 & 21.8 & 110 & 19.7 & 30.4 & 499 & 15.5 & 26.1 & 0.224 \\
\hline & $\mathrm{p}$-value & & 0.004 & & & 0.676 & & & 0.055 & & & 0.002 & & \\
\hline
\end{tabular}


OS by modified Glasgow Prognostic Score

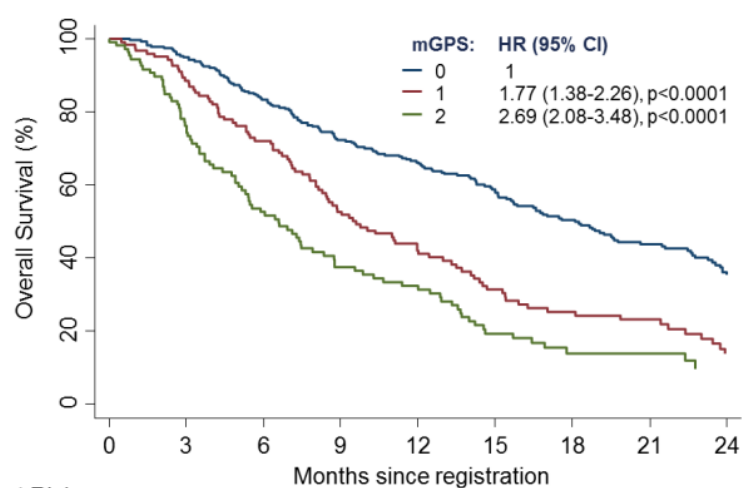

Number at Risk:

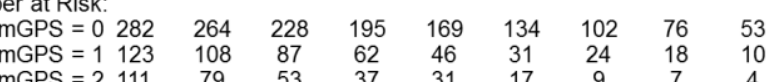

PFS by modified Glasgow Prognostic Score

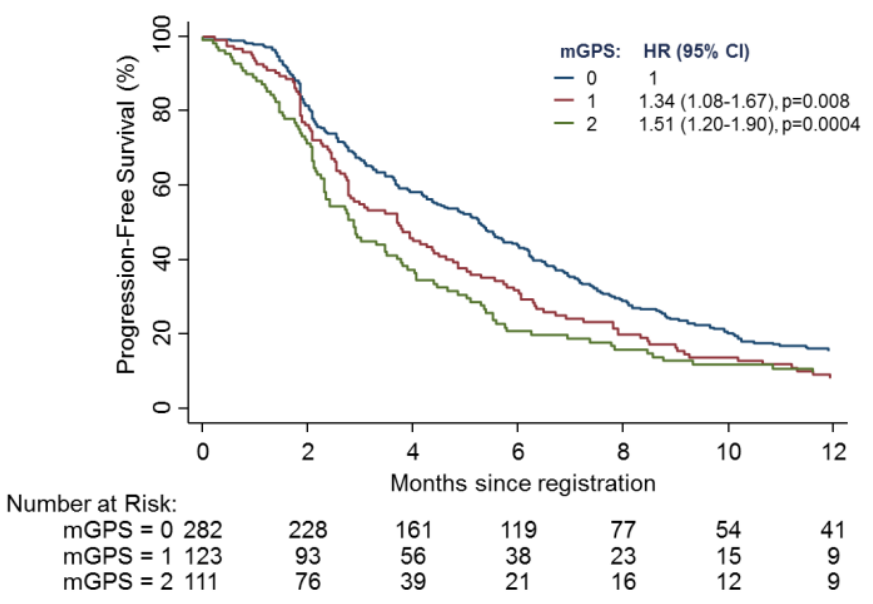

Figure 1. Kaplan-Meier curves showing OS and PFS by modified Glasgow Prognostic Score 